\title{
Erratum to: Clinical implication of ocular torsion in peripheral vestibulopathy
}

\author{
Jin Woong Choi $\cdot$ Seong Il Kang $\cdot$ Ji Hye Rhee $\cdot$ \\ Byung Yoon Choi $\cdot \mathrm{Ji}$-Soo Kim $\cdot$ Ja-Won Koo
}

Published online: 18 April 2014

(C) Springer-Verlag Berlin Heidelberg 2014

\section{Erratum to: Eur Arch Otorhinolaryngol}

DOI 10.1007/s00405-014-2952-3

One of the co-author's name was published incorrectly as Byeong Yoon Choi. The correct spelling is Byung Yoon Choi.

The online version of the original article can be found under doi:10.1007/s00405-014-2952-3.

J. W. Choi

Department of Otolaryngology-Head and Neck Surgery,

Chungnam National University School of Medicine, Daejeon,

South Korea

S. I. Kang · J. H. Rhee $\cdot$ B. Y. Choi · J.-W. Koo $(\bowtie)$

Department of Otorhinolaryngology, Seoul National

University, College of Medicine, Seoul National University

Bundang Hospital, 166 Gumi-ro, Bundang-gu, Seongnam,

Gyeonggi-do 463-707, South Korea

e-mail: jwkoo99@snu.ac.kr

B. Y. Choi · J.-W. Koo

Research Center for Sensory Organs, Seoul National

University Medical Research Center, Seoul, South Korea

J.-S. Kim

Department of Neurology, Seoul National University,

Bundang Hospital, Seongnam, South Korea 